Abanico Veterinario. Enero-Diciembre 2020; 10(1):1-11. http://dx.doi.org/10.21929/abavet2020.6 Artículo Original. Recibido: 16/01/2020. Aceptado: 25/04/2020. Publicado: 30/04/2020.

\title{
Utilización de subproductos de naranja (Citrus sinensis var. valencia) en la alimentación para rumiantes
}

\author{
Orange by-products use (Citrus sinensis var.valencia) in ruminants feed
Cabrera-Núñez Amalia $\stackrel{\mathbb{D}}{\mathrm{D}}$, Lammoglia-Villagómez Miguel $\underline{\mathrm{ID}}$, Martínez-Sánchez

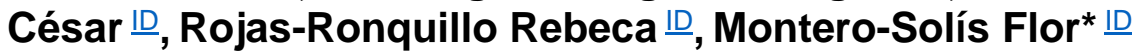

Facultad de Ciencias Biológicas y Agropecuarias, Universidad Veracruzana. Tuxpan, México. *Autora responsable y de correspondencia: Montero-Solís Flor. Facultad de Ciencias Biológicas y Agropecuarias de la Universidad Veracruzana. Carretera Tuxpan-Tampico Km. 7.5, Colonia Universitaria, Tuxpan, Veracruz, México, CP 92890. 017838344350 ext. 46109. Tuxpan, Veracruz. amacabrera@uv.mx, malammoglia@uv.mx, cesamartinez@uv.mx, rebrojas@uv.mx, flmontero@uv.mx.

\begin{abstract}
RESUMEN
Este trabajo tuvo como objetivo elaborar un concentrado alimenticio con subproductos de naranja valencia Citrus sinensis var. valencia como suplemento para toretes de engorda en el norte del estado de Veracruz. Se desarrolló en una Unidad de Producción Pecuaria (UPP) del Municipio de Álamo-Temapache, Veracruz. Geográficamente localizado entre los meridianos Latitud norte: $20^{\circ} 55^{\prime} 19^{\prime \prime}$, Latitud oeste $97^{\circ} 41^{\prime} 44^{\prime \prime}$. La localidad se encuentra a una altura de $40 \mathrm{msnm}$ y temperatura media anual de $19-36^{\circ} \mathrm{C}$. Se evaluaron, 40 toretes Cebú x Suizo, con un peso aproximado de $300 \mathrm{Kg}$, que fueron estabulados y asignados bajo un diseño completamente aleatorio en cuatro tratamientos y diez repeticiones $(n=10 \mathrm{c} / \mathrm{u})$. T1 (testigo) forraje fresco; T2: concentrado formulado a base de bagazo de naranja + forraje fresco; T3: concentrado formulado con cascara de naranja + forraje fresco; T4: concentrado formulado a base de ensilado de naranja + forraje fresco. El alimento se ofreció dos veces al día a libre acceso considerando el 3.0\% (NRC) del peso vivo durante los 90 días del experimento, realizándose dos pesajes al inicio y final. El promedio de ganancia diaria $(P<0.05)$ fue $2.97 \mathrm{~kg} /$ animal y rendimiento en canal fría de $55.3 \%$, para los animales que recibieron una suplementación de subproductos a base de silo naranja (T4) al 13.3\% PC, promovió un mayor rendimiento en los bovinos de engorda.
\end{abstract}

Palabras clave: naranja valencia, subproductos, rendimiento, bovinos y silo.

\begin{abstract}
This study was aimed to elaborate a bull fattening supplement with Valencia orange by-products (Citrus sinensis var. valencia) in the north of Veracruz state. It was performed in a Livestock Production Unit (UPP) from the Municipality of Álamo-Temapache, Veracruz. Geographically located at North latitude: $20^{\circ} 55^{\prime} 19$ ", West latitude $97^{\circ} 41^{\prime} 44$. This place is found at 40 meters height above sea level with an average annual temperature of $19-36^{\circ}$ C. 40 Zebu x Swiss bulls, with $300 \mathrm{~kg}$ weight approximately, were housed and assigned under a completely randomized design in four treatments, and ten repetitions $(n=10)$. T1 (control) fresh forage; T2: formulated concentrate based on orange bagasse + fresh forage; T3: concentrate formulated with orange peel + fresh forage; T4: concentrate formulated with orange silage + fresh forage. Food was offered twice a day "ad libitum" considering $3.0 \%$ (NRC) of the live weight during the 90 days of the experiment, with two weightings at the beginning and at the end. The average daily gain $(P<0.05)$ was $2.97 \mathrm{~kg} /$ animal and the cold carcass yield was 55.3\%, to the animals that received a by-product supplementation based on orange silo (T4) $13.3 \%$ PC it promoted higher performance in fattening cattle.

Keywords: orange valence, by-products, yield, cattle, silo.
\end{abstract}




\section{INTRODUCCIÓN}

En los últimos años y debido a la preocupación ambiental de la sociedad, la agroindustria debe ser sensible a los temas ambientales, procurando el desarrollo creciente de una conciencia social, que obliga a no producir a costa del planeta, sino de una manera sostenible. Por lo anterior, se han estudiado las características nutricionales de los subproductos de la agroindustria, para la elaboración de raciones alimenticias para animales (Escorza et al., 2019). La mejor estrategia para alcanzar una mayor productividad consiste en establecer un programa de complementación alimenticia, utilizando los recursos materiales, humanos y financieros disponibles. Dado que la decisión de complementar representa un costo adicional al productor; se debe considerar la utilización de aquellos complementos que brinden la máxima respuesta productiva y económica (Cury et al., 2017).

Las industrias que procesan los productos agropecuarios, deben encontrar un destino final para los subproductos, que cumplan con dos requisitos mínimos; que no sea contaminante del ambiente y que los costos de procesamiento y transportación sean cubiertos por el precio de venta de los mismos y no generen costos adicionales a la producción (Bisang, 2017). Entre los distintos subproductos, hay una alta variabilidad en la composición química disponible; algunos pueden ser muy regulares en su composición, porque los procesos industriales que los generan están altamente controlados. Mientras que otros pueden presentar una variación importante, por lo que es necesario conocer los aportes nutricionales al momento de formular las raciones (Berenguer, 2000).

Una vez determinados los aportes a realizar por medio de la suplementación, se debe tener en cuenta que muchos subproductos pueden aportar simultáneamente energía y proteína (Lagos y Castro, 2019); otros en cambio, pueden ser carentes de algún nutriente. Es importante destacar que la alimentación animal a partir de subproductos son sustitutos y son considerados además como alternativas que pueden elegir los productores, acorde a sus posibilidades económicas y distribución geográfica, con la finalidad de incrementar las ganancias de peso, conversión alimenticia y una mayor rentabilidad económica (Campos y Arce, 2016).

Los cítricos se cultivan ampliamente en áreas subtropicales o de clima mediterráneo, y en menor escala en zonas tropicales. La mayor parte de los incrementos en la producción se ha debido al crecimiento de las superficies del cultivo de naranja, y a que se ha elevado la productividad, gracias al mejoramiento de las prácticas de explotación; mediante la aplicación de fertilizantes y riego, el control de plagas y enfermedades, injerto de árboles, sustitución de huertos mal localizados y la siembra de las variedades con mayor resistencia (Hernández, 2015). 
La naranja valencia (Citrus sinensis var.valencia), es uno de los productos que más se consumen en todo el mundo, y un poco más del 10\% de la producción total, aproximadamente 6,5 millones de toneladas, se exporta a otros países, como: Francia, Reino Unido, Alemania, Rusia, Arabia Saudita, entre otros. Es uno de los productos de mayor consumo mundial y un poco más del $10 \%$ de la producción total, (Gómez et al., 2019). En México la producción de cítricos se destina al consumo doméstico (88\%), y el resto es exportado principalmente a Estados Unidos (Valencia y Duana, 2019).

Las naranjas se posicionan en la quinta posición de las listas de productos agrícolas más producidos o exportados, superadas solamente por las bananas, manzanas, tomates y las cebollas, en cuanto al comercio internacional. México se encuentra en el lugar veinticinco con 19,449 dólares y contribuye con $0.4 \%$ de las exportaciones totales (Triana et al., 2014).

El estado de Veracruz cuenta con casi $50 \%$ de la superficie cosechada del país (Barrón y Hernández, 2014) y produce el 50 \% de la naranja de jugo (SADER, 2018) a nivel nacional. El municipio de Álamo, localizado al norte del estado de Veracruz, es considerado como la capital de la naranja; produciendo variedad de valencia tardía, valencia súper tardía y valencia temprana; así como las de mesa (zarzuma, mónica y mandarinas), entre otras. Además cuenta con jugueras que procesan la naranja y que añaden valor a los subproductos derivados (Sarah et al., 2018), como la pulpa o desecho de cítricos; integrado por el jugo, bagazo, semillas y la cáscara (Espinoza et al., 2019) para producir ensilado.

El bagazo fresco, por su alto contenido de agua y su potencial contaminante del ambiente, genera un problema a nivel de las plantas industriales, pero ofrece al mismo tiempo una oportunidad de alimentación suplementaria para rumiantes, especialmente los bovinos (Bermúdez et al., 2015).

Derivado de la alta producción de naranja en la zona, estos subproductos llegan a ser suministrados por los dueños de los ranchos ganaderos en épocas de alta producción. Gran parte de estos cítricos son destinados a la producción de jugo, obteniendo anualmente unas 70,000 toneladas de pulpa que son descartadas por la juguera.

Por lo anterior y debido a la alta demanda de subproductos de la naranja, este trabajo tuvo como objetivo elaborar un concentrado alimenticio con subproductos de naranja valencia (Citrus sinensis var. valencia) como suplemento para toretes de engorda en el norte del estado de Veracruz. 


\section{MATERIAL Y MÉTODOS}

\section{Lugar experimental}

Esta investigación se desarrolló en un rancho ganadero en el municipio de ÁlamoTemapache, Veracruz; localizado en los meridianos Latitud Norte $20^{\circ} 55^{\prime} 19^{\prime \prime}$ y una Longitud Oeste $97^{\circ} 41^{\prime} 44^{\prime \prime}$; a una altura de 40 msnm y temperatura ambiental 19 a $38^{\circ} \mathrm{C}$ (Solano, 2006).

\section{Tratamientos}

Se emplearon 40 toretes Cebú x suizo, con un peso aproximado de $300 \mathrm{~kg}$. asignados bajo un diseño completamente aleatorio en cuatro tratamientos y diez repeticiones por tratamiento.

\section{Determinación de valores nutricionales de la cáscara de naranja}

Los valores nutricionales (proteína cruda \%, extracto etéreo \%, cenizas \%, fibra cruda \% (Van Soest y Wine, 1968), extracto libre de nitrógeno \%, total de nutrientes digestibles (Mcal) y energía neta de producción (Mcal) en la cáscara de naranja; se analizaron previo a la elaboración de concentrados en el Laboratorio de Bromatología Animal de la Facultad de Ciencias Biológicas y Agropecuarias de la Universidad Veracruzana, campus Tuxpan.

\section{Manejo de rumiantes}

El manejo de los animales estabulados, consistió en vacunación, desparasitación, identificación con arete y aplicación de vitaminas A, D y E, por vía intramuscular. El suministro del alimento concentrado fue ofrecido dos veces al día, considerando el 3\% del peso vivo del torete (NRC, 2007) durante los 90 días que duró el experimento. Los tratamientos fueron forraje fresco ( $\mathrm{T} 1$ testigo), concentrado formulado con base en bagazo de naranja + forraje fresco (T2), concentrado formulado a base cáscara de naranja + forraje fresco (T3) y concentrado formulado a base de ensilado de naranja + forraje fresco $(\mathrm{T} 4)$.

\section{Elaboración de concentrados}

Para llevar a cabo la elaboración del concentrado alimenticio, para los tratamientos T2 y T3, fue necesario recolectar subproductos que eran desechados como cáscara y bagazo de naranja en las jugueras de Álamo, Veracruz. En relación con el tratamiento T4, se elaboró un silo tipo pastel, ubicado en un sitio elevado y con ligera pendiente, construyendo un borde alrededor para evitar la entrada de agua, como único ingrediente; las naranjas se picaron en trozos pequeños de $3-6 \mathrm{~cm}$; las que se acumularon por capas con $20 \mathrm{~cm}$ de espesor y se apisonó con un tractor por encima. Posteriormente se cubrió con plástico de polietileno, y sobre ésta una capa uniforme de $10-15 \mathrm{~cm}$ de tierra, con seis metros de ancho y uno de alto; el cual se utilizó un mes después para descubrir solamente la cantidad a proporcionar por día. 
Para facilitar el mezclado de los ingredientes, se agregó sobre lonas extendidas y por separado cada uno de los tratamientos: T2: $50 \mathrm{~kg}$. bagazo de naranja; T3: $50 \mathrm{~kg}$. cáscara de naranja y T4: $50 \mathrm{~kg}$. silo de naranja. Con lo anterior se elaboró el concentrado, agregando maíz quebrado (25 kg.), sorgo molido (20 kg.), vitaminas y minerales (5 kg.). Los ingredientes se mezclaron diaria y cuidadosamente, para tener un alimento homogéneo en las distintas raciones para ser suministrado mañana y tarde al ganado (tablas 1,2 y 3 ).

Tabla 1. Ingredientes utilizados para la elaboración de concentrado alimenticio a base de bagazo naranja

\begin{tabular}{lccc}
\hline \multicolumn{1}{c}{ Ingredientes } & $\mathrm{kg}$ & $\mathrm{PC} \%$ & TOTAL PC\% \\
\hline Bagazo de naranja & 50 & 10 & 5.10 \\
Maíz quebrado & 25 & 12 & 2.75 \\
Sorgo molido & 20 & 12 & 2.45 \\
Vitaminas y minerales & 5 & - & - \\
& & & 10.3 \\
TOTAL & 100 & &
\end{tabular}

Tabla 2. Ingredientes utilizados para la elaboración de concentrado alimenticio a base de cáscara de naranja

\begin{tabular}{lccc}
\hline \multicolumn{1}{c}{ Ingredientes } & $\mathrm{kg}$ & $\mathrm{PC} \%$ & TOTAL PC\% \\
\hline Cáscara de naranja & 50 & 10.0 & 5.10 \\
& & & \\
Maíz quebrado & 25 & 12 & 2.75 \\
Sorgo molido & 20 & 12 & 2.45 \\
Vitaminas y & 5 & - & - \\
minerales & & & 10.3 \\
TOTAL & 100 & &
\end{tabular}

PC (Proteína Cruda).

Tabla 3. Ingredientes utilizados para la elaboración de concentrado alimenticio a base de silo de naranja

\begin{tabular}{llll}
\hline Ingredientes & $\mathrm{Kg}$ & $\mathrm{PC} \%$ & TOTAL PC\% \\
\hline Silo de naranja & 50 & 16.0 & 8.10 \\
Maíz quebrado & 25 & 12 & 2.75 \\
Sorgo molido & 20 & 12 & 2.45 \\
Vitaminas y & 5 & - & \\
minerales & & & 13.3 \\
TOTAL & 100 & & \\
\hline PC (Proteína Cruda). & &
\end{tabular}

\section{Estimación de peso}

Los cambios en el peso de los animales fueron registrados al inicio y término del estudio (90 días). Una vez finalizadas las evaluaciones los animales fueron sacrificados en un rastro frigorífico Tipo Inspección Federal (TIF), determinándose el rendimiento de la canal fría (RCF).

\section{Análisis de datos}

Se realizó un análisis de varianza y prueba de Duncan para comparar las medias de tratamientos $(P<0.05)$, con el paquete estadístico SPSS (SPSS, versión 10). 


\section{RESULTADOS}

En cuanto a las recomendaciones nutricionales indicadas por la National Research Council (NRC, 2001), los valores entre 10-18\% de Proteína Cruda (PC) y $65 \%$ de Total de Nutrientes Digestibles (TND), son considerados deseables (tabla 4). Por lo que en esta investigación se cumplió con estos parámetros, destinados a los toretes estabulados de 300 kg., con valores de $10.0-13.0$ \% PC proporcionada en la ración.

El comportamiento productivo de los animales en los distintos tratamientos, relacionados con la ganancia de peso $(\mathrm{kg} / \mathrm{día})$ fue significativamente diferente $(P<0.05)$, entre los tratamientos (T2, T3 y T4), con respecto al control (2.66, 2.70 y $2.97 \mathrm{~kg}$.) (tabla 5).

Tabla 4. Valor nutrimental de los subproductos de naranja

\begin{tabular}{lcccc}
\hline \multicolumn{1}{c}{ Indicador } & $\begin{array}{c}\text { Bagazo de } \\
\text { naranja }\end{array}$ & $\begin{array}{c}\text { Cáscara de } \\
\text { naranja }\end{array}$ & $\begin{array}{c}\text { Ensilado de } \\
\text { naranja }\end{array}$ & $\begin{array}{c}\text { Forraje } \\
\text { Fresco }\end{array}$ \\
\hline Proteína Cruda \% & 10.0 & 10.0 & 16.0 & 5.67 \\
Extracto Etéreo \% & 3.20 & 4.96 & 9.22 & 3.79 \\
Cenizas \% & 3.30 & 7.92 & 7.93 & 3.02 \\
Fibra Cruda \% & 14.92 & 30.80 & 33.40 & 36.43 \\
Extracto libre de Nitrógeno \% & 64.51 & 67.18 & 77.58 & 56.78 \\
Total de nutrientes digestibles (Mcal) & 60.39 & 63.78 & 64.56 & 55.67 \\
Energía Neta de Producción (Mcal) & 22.60 & 26.87 & 27.89 & 29.30 \\
\hline
\end{tabular}

PC (Proteína Cruda). Mcal (megacalorías).

Tabla 5. Niveles productivos con subproductos de naranja

\begin{tabular}{|c|c|c|c|c|c|c|c|c|}
\hline & T1 & SD & T2 & SD & T3 & SD & $\mathrm{T} 4$ & SD \\
\hline Peso inicial $(\mathrm{kg})$ & $300.1 \mathrm{a}$ & 33.11 & $300.2 \mathrm{a}$ & 33.11 & $300.1 \mathrm{a}$ & 32.99 & $300.1 \mathrm{a}$ & 33.11 \\
\hline Peso final $(\mathrm{kg})$ & $439.8 \mathrm{a}$ & 28.19 & $540.3 \mathrm{~b}$ & 28.19 & $543.5 \mathrm{~b}$ & 11.00 & $567.5 \mathrm{a}$ & 6.80 \\
\hline $\begin{array}{l}\mathrm{Kg} \text { ganados/animal } \\
90 \text { días/grupo }\end{array}$ & 139.7 a & - & $240.1 \mathrm{~b}$ & - & 243.4 b & - & $267.4 \mathrm{~b}$ & - \\
\hline $\begin{array}{l}\text { Ganancia de } \\
\text { peso/día (kg) }\end{array}$ & $1.55 \mathrm{a}$ & 0.32 & $2.66 \mathrm{~b}$ & 0.53 & $2.70 \mathrm{~b}$ & 0.40 & $2.97 \mathrm{~b}$ & 0.38 \\
\hline $\begin{array}{l}\text { Consumo del } \\
\text { concentrado (kg) }\end{array}$ & - & - & $16.20 \mathrm{~b}$ & - & $16.30 \mathrm{~b}$ & - & $17.02 \mathrm{~b}$ & - \\
\hline $\begin{array}{l}\text { Consumo de } \\
\text { energía } \\
\text { metabolizante } \\
\text { (Mcal) }\end{array}$ & $10.30 \mathrm{a}$ & - & 27.89 b & - & 27.92 b & - & 27.94 b & - \\
\hline $\begin{array}{l}\text { Rendimiento de } \\
\text { canal fría (\%) }\end{array}$ & $50.0 \mathrm{a}$ & - & $54.5 \mathrm{~b}$ & - & $54.8 \quad b$ & - & $55.3 \mathrm{~b}$ & - \\
\hline
\end{tabular}




\section{DISCUSIÓN}

La pulpa de naranja proveniente de la juguera, presentó un valor proteico de 10 - $13 \%$; estos valores de proteína cruda (PC) podrían llegar a incrementarse y mejorar la ganancia diaria de peso, mediante la adición de proteína de mediana calidad, como la urea, gallinaza, pollinaza o alguna proteína de sobrepaso; como pasta de soya, harinolina, semilla de algodón, entre otros (Lagos y Castro, 2019). En raciones alimenticias con subproductos de cítricos para rumiantes (Hernández et al., 2015), se ha observado que la ganancia de peso, calidad de la canal y el contenido de grasa de leche se incrementa, lo que dio resultados similares con el suministro de cereales en la ración alimenticia proporcionada en este estudio. La diferencia en el consumo de alimento dio como resultado que los animales del T1, consumieran $12.6 \mathrm{Mcal}$; mientras que los animales que consumieron subproductos de naranja (T2, T3 y T4), ingirieron entre 27.89 a 29.9 Mcal de energía neta de producción/día (tabla 5).

Se destaca, que los animales que recibieron sólo nutrientes energéticos presentaron mayores kilogramos ganados/animal a la adición del subproducto de naranja (240.1, 243.4 y $267.4 \mathrm{~kg}$, para T2, T3 y T4, respectivamente), en comparación con los tratamientos suplementados únicamente con fibra (T1), los que tuvieron una respuesta significativamente inferior $(P<0.05)$. Asimismo, es notable el rendimiento de la canal fría (55.3\%), el cual fue significativamente superior en los animales suplementados con silo de maíz. Lo anterior está relacionado con lo reportado por Cury et al. (2017), quienes reconocen que la producción diaria por animal está determinada por la combinación de efectos entre la disponibilidad y calidad del alimento (suplementación energético proteica); así como por el apetito y el potencial genético del animal.

Actualmente, en el campo de la nutrición de los bovinos productores de carne, no sólo es importante la proteína, ya que el nivel y calidad de la proteína es fundamental en la respuesta productiva de los animales, y es la clave para lograr las mayores utilidades en cualquier unidad de producción ganadera, bajo condiciones de confinamiento y el comportamiento productivo de los animales en los distintos tratamientos.

En relación con la ganancia total de peso/día, hubo incrementos de 2.66, 2.70, $2.97 \mathrm{~kg}$. para T2, T3 y T4, en los toretes que recibieron subproductos de naranja con respecto al tratamiento testigo. La diferencia en el consumo de alimento (T1: pasto) fue de 10.30 Mcal de energía metabolizable/día. Mientras que los toretes suplementados con subproductos de naranja, consumieron 27.89, 27.92 y 27.94 Mcal (T2, T3, T4, respectivamente); equivalente a una diferencia de $40 \%$, que se refleja en la misma proporción sobre la ganancia de peso vivo entre los tratamientos. Estas diferencias se debieron a un mejor aporte nutricional y al efecto que tiene en el rumen la inclusión de una fuente de energía para la producción de los ácidos grasos volátiles (AGV), como el ácido acético, ácido propiónico, butírico y valérico, imprescindibles sobre el consumo de 
alimento, la ganancia diaria de peso vivo e incremento de la producción de carne y conformación (Jarvis y Moore, 2010).

En relación con la calidad del silo de naranja (T4), se obtuvo un ensilaje de buena calidad; debido a su color verde amarillento y café claro, un olor a fruta madura, ligeramente ácido y una textura firme, sin deformarse al presionar con los dedos; lo cual indica que el manejo adecuado del silo aumentó las propiedades nutritivas y organolépticas del alimento. Cabe destacar que la cantidad de alimento ofrecido a los animales del tratamiento 2, 3 y 4, fue totalmente consumida; esto debido a la palatabilidad del subproducto de naranja, lo cual quedó demostrado al no quedar residuos dentro de los comederos. Por consiguiente, se podría atribuir que el consumo de materia seca es total en todos los tratamientos, excepto en el T1 (forraje fresco), en éste se observó que, aunque fue consumido no fue atractivo, en comparación a los subproductos de naranja. Lo anterior puede deberse al incremento de los azúcares digestibles que estimulan la palatabilidad, en comparación a los forrajes verdes o secos, al poseer mayor cantidad de carbohidratos. Las bacterias ácido lácticas generan mayor cantidad de compuestos bioquímicos que enriquecen el sustrato, mejorando las condiciones de sabor y estimulando sensorialmente a través de su olor característico a ácido láctico y los órganos sensitivos del olfato animal (Wadhwa et al., 2015).

Los resultados del presente trabajo fueron similares a los reportados por Triana et al. (2014), quienes trabajaron con subproductos de naranja valencia, apreciando en el silo un color marrón claro y mostaza claro a través de los tiempos de fermentación. El silo elaborado en este estudio cumple con las características organolépticas asociadas con ensilajes de alta calidad; en este caso un olor a vino fermentado aromático, dulzón, agradable, que caracteriza al ácido láctico. Esto podría atribuirse a que las cáscaras de naranja además de la fragancia natural de los aceites esenciales, poseen ácidos que generan un olor agradable que acompañado del aumento de bacterias lácticas en el proceso de fermentación, le confirieron al ensilaje un olor ligeramente agradable.

Actualmente, en el campo de la nutrición de los bovinos productores de carne, no sólo es importante la proteína, ya que el nivel y calidad de la energía es fundamental en la respuesta productiva de los animales y es la clave para lograr las mayores utilidades en cualquier sistema de producción ganadera, bajo condiciones de confinamiento. Es importante destacar que la energía provee al organismo la capacidad de realizar trabajo. En raciones para el ganado de engorda, la energía se requiere para actividades como crecimiento y mantenimiento; por lo que la energía es un nutriente requerido por el ganado en grandes cantidades. Las fuentes primarias de energía en los forrajes son la celulosa y la hemicelulosa, y en los granos lo es el almidón. Las grasas y los aceites tienen un mayor contenido de energía, pero usualmente se adicionan en pequeñas cantidades en la dieta. 


\section{CONCLUSIONES}

Fue muy notable que los toretes estabulados que recibieron los subproductos de silo de naranja presentaron una ganancia diaria de peso de $2.97 \mathrm{~kg}$. La ganancia de peso acumulada a los 90 días fue de $267.4 \mathrm{~kg}$. y el tratamiento para los toretes con forraje fresco de pastura de estrella de áfrica, resultó ser inferior, con $139.7 \mathrm{~kg}$. El bajo precio y fácil elaboración de concentrados con subproductos de naranja es atractivo y una opción viable, como complemento para rumiantes en épocas de estiaje (sequía e invierno), dada la disponibilidad del cítrico en el norte del estado de Veracruz.

\section{LITERATURA CITADA}

BERENGUER JM, Corraliza JA. 2000. Preocupación ambiental y comportamientos ecológicos. 12 (3): 325-329. Psicothema ISSN: 0214 - 9915. https://www.researchgate.net/publication/28112416_Preocupacion_ambiental_y_compo rtamientos_ecologicos.

BISANG R. 2017. Problemas actuales y perspectivas futuras de la producción y comercialización de granos / Roberto Bisang; José Pierri; compilado por Roberto Bisang; José Pierri. - 1a Edición. Ciudad Autónoma de Buenos Aires: Universidad de Buenos Aires. Facultad de Ciencias Económicas. Libro digital. ISBN: 978-950-29-1670-5. http://bibliotecadigital.econ.uba.ar/download/libros/Bisang-Pierri_Problemas-actuales-yperspectivas-futuras-2017.pdf.

BARRÓN PMA, HERNÁNDEZ TJM. 2014. Productores y cortadores de naranja, una relación fallida. El municipio de Álamo Temapache, Veracruz. Rev Mex Cienc Agríc. 5(6):1097-1109. ISSN: 2007-0934.

http://www.scielo.org.mx/scielo.php?script=sci_arttext\&pid=S200709342014000600015.

BERMÚDEZ LJA, Melo CEP, Estrada AJ. 2015. Evaluación de ensilaje de naranja entera (Citrus sinensis) como alternativa de suplementación en bovinos. Vet y Zoot. 9(2):38-53. ISSN:2011-5415. http://dx.doi.org/10.17151/vetzo.2015.9.2.4.

https://www.researchgate.net/publication/316230044_Evaluacion_de_ensilaje_de_naran ja_entera_Citrus_sinensis_como_alternativa_de_suplementacion_en_bovinos.

CAMPOS GCM, Arce VJ. 2016. Sustitutos de maíz utilizados en la alimentación animal en Costa Rica. Nutr Anim Trop. 10(2):91-113. http://dx.doi.org/10.15517/nat.v10i2.27327.

CURY R, Katia, Aguas MY, Martínez MA, Olivero V, Rafael, Chams Ch, Linda. 2017. Residuos agroindustriales su impacto, manejo y aprovechamiento. Rev. Colombiana de Cienc Anim Recia. 9 (supl):122-132 ISSN: 2027-4297. https://doi.org/10.24188/recia.v9.nS.2017.530.

ESCORZA MM, Gibran AG, García EJ, Ayala MM, Zepeda BA, Soto SS. 2019. Comportamiento productivo y calidad de la carne de conejos que consumieron 
desperdicio de galleta. Abanico Veterinario. 9 (1): 2-4. ISSN: 2448-6132. http://dx.doi.org/10.21929/abavet2019.910.

ESPINOZA ZA, Orozco BG, Vázquez LY, Romo RJ, Escalera VF, Martínez GV. 2019. Una revisión sobre la pulpa de naranja: cantidad, composición y usos. Abanico Agroforestal. Enero-Diciembre. 1 (1):1-14. ISSN: ISSN 2594-1992. https://abanicoacademico.mx/revistasabanico/index.php/abanico-

agroforestal/article/view/208

GÓMEZ MR, CAAMAL CI, FERNÁNDEZ VG. 2019. Análisis de la distribución y comportamiento de los indicadores del comercio mundial de la naranja. Anales Iberoamericanos de Economía Aplicada (Asepetl). 1: 67-77. ISSN: 2659-6369. http://iberoamerica.asepelt.org/media/attachments/2019/03/13/aibea-i.pdf.

HERNÁNDEZ MA, González RA, Rojo R, Sánchez DF, Salvador A, Vázquez AJF. 2015. Producción y composición de la leche de cabras alimentadas con diferentes inclusiones de cáscara de naranja deshidratada. Rev Cient. 25(4): 324-329. https://www.researchgate.net/publication/280938840.

HERNÁNDEZ MA, García SJ, Omaña JM, Hernández RO, Cruz GB. 2015. Análisis competitivo de sistemas de producción de naranja (Citrus sinensis) en Nuevo León, México. Rev Agroproductividad. 8 (2): 52-59. ISSN: 0188-7394. https://www.colpos.mx/wb_pdf/Agroproductividad/2015/Agroproductividad_II_2015.pdf\# page $=54$.

JARVIS GN, Moore ERB. 2010. Handbook of Hydrocarbon and Lipid Microbiology, DOI 10.1007/978-3-540-77587-4_163, \# Springer-Verlag Berlin Heidelberg. 2246-2255 p. https://www.researchgate.net/profile/Edward_Moore/publication/274735522_Lipid_Meta bolism_and_the_Rumen_Microbial_Ecosystem/links/55293fa10cf2779ab7907947/LipidMetabolism-and-the-Rumen-Microbial-Ecosystem.pdf.

LAGOS BE, Castro RE. 2019. Caña de azúcar y subproductos de la agroindustria azucarera en la alimentación de rumiantes. Agron. Mesoam. 30(3): 917-934. ISSN 22153608.doi:10.15517/am.v30i3.34668.https://www.researchgate.net/publication/33558681 3_Cana_de_azucar_y_subproductos_de_la_agroindustria_azucarera_en_la_alimentaci on_de_rumiantes_1_Sugar_cane_and_by-products_of_the_sugar_agroindustry_in_ruminant_feeding_A_review.

NATIONAL RESEARCH COUNCIL (NRC). 2001. Nutrient requeriments of dairy cattle. Editorial National Academy Press, Washington DC. USA. 405 p. ISBN: 978-0-309-069977. https://doi.org/10.17226/9825.

SADER (SECRETARIA DE AGRICULTURA Y DESARROLLO RURAL). 2018. Delegación SADER Veracruz.

https://www.gob.mx/agricultura/veracruz/articulos/aporta-veracruz-el-50-de-laproduccion-nacional-de-naranja?idiom=es. 
SARAH S, Rahman WW, Majid RA. 2018. Optimization of Pineapple Leaf Fibre Extraction Methods and Their Biodegradabilities for Soil Cover Application. $J$ of polymers and the Environ. 26:319. ISSN: 1572-8900. https://doi.org/10.1007/s10924-017-0942-4.

SOLANO G, Cobos V, Fernández JL, Ramírez RC. 2001. Elaboración y evaluación de subproductos industriales para la alimentación animal. Rev Cubana de Ciencia Agrícola. 35 (4):345-348. ISSN: 0034-7485.

https://www.redalyc.org/articulo.oa?id=193018246005.

TRIANA E, Leal FC, Lizcano H. 2014. Evaluación de ensilaje a partir de dos subproductos agroindustriales (cascara de naranja y plátano de rechazo) para alimentación de ganado bovino. Rev alim Hoy. 22 (31):33-45 ISSN: 2017-291X. https://acta.org.co/acta_sites/alimentoshoy/index.php/hoy/article/view/254/238.

VALENCIA SK, Duana AD. 2019. Los cítricos en México: análisis de eficiencia técnica. Análisis económico 34(87):269-283. ISSN: 0185-3937, e- ISSN: 2448-6655. http://analisiseconomico.azc.uam.mx/index.php/rae/article/view/483/349.

VAN Soest PJ, Wine RH. 1968. Determination of lignin and cellulose in aciddetergent fibre with permanganate. $J$ of the Association of Official Agricultural Chemists. 51:780. https://www.scirp.org/(S(Iz5mqp453edsnp55rrgjct55)/reference/ReferencesPapers.aspx ?ReferencelD=1496671.

WADHWA M, Bakshi MPS, Makkar HPS. 2016. Wastes to worth: value added products from fruit and vegetable wastes. CAB Reviews. 10(43):1-25. ISSN: 1749-8848. http://dx.doi.org/10.1079/PAVSNNR201510043.https://www.deepdyve.com//p/centre-foragricultural-bioscience-international/wastes-to-worth-value-added-products-from-fruitand-vegetable-wastes-7UVj84CRyB.

SOLANO LF. 2006. Monografía de Álamo. 134 p. https://www.alamotemapache.gob.mx/goba/index.php/historia/1559monografia?showall=1.

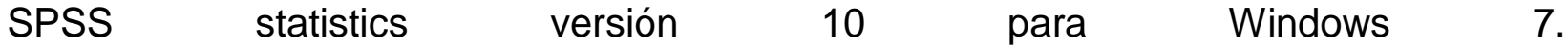
https://softadvice.informer.com/Free_Download_Spss_10_For_Windows_7.html. 